\title{
Effects of partial crystallinity and quenched-in defects on corrosion of a rapidly solidified $\mathrm{Ti}-\mathrm{Cu}$ alloy
}

\author{
R S DUTTA* and G K DEY \\ Materials Science Division, Bhabha Atomic Research Centre, Mumbai 400 085, India
}

MS received 27 February 2003

\begin{abstract}
Rapid solidification by planar flow casting has been found to have introduced deficiencies, viz. partial crystallinity, air pockets and compositional difference in the ribbons of rapidly solidified $\mathbf{T i}_{42.9} \mathrm{Cu}_{57 \cdot 1}$ alloy. In order to investigate the effects of these deficiencies on the corrosion of rapidly solidified $\mathrm{Ti}_{42.9} \mathrm{Cu}_{57.1}$ alloy ribbons, electrochemical behaviour of alloy ribbons has been investigated in the acidic chloride environments at room temperature by taking into consideration each side of the alloy ribbon separately. The alloy displayed passivity followed by pitting corrosion. In the as-solidified condition, air pockets appear to be the most detrimental defect from the viewpoint of corrosion resistance of the alloy ribbons.
\end{abstract}

Keywords. Rapidly solidified; partial crystallinity; passivity; pitting.

\section{Introduction}

Amorphous materials are more corrosion-resistant than crystalline ones because these are devoid of crystalline defects such as grain boundaries, vacancies and dislocations and possess good compositional homogeneity. Although numerous studies have been carried out on the corrosion aspects of various amorphous alloys, binary $\mathrm{Ti}-\mathrm{Cu}$ amorphous alloys have received very little attention (Naka et al 1978; Dutta et al 2001). The corrosion behaviour of equiatomic amorphous $\mathrm{Ti}-\mathrm{Cu}$ alloy has been compared with its crystalline counterpart in various environments at room temperature $(R T)$ by some researchers (Naka et al 1978). According to Naka et al (1978), the corrosion resistance of equiatomic amorphous $\mathrm{Ti}-\mathrm{Cu}$ alloy is superior to its crystalline counterpart and the superiority is believed to be attributable to higher chemical homogeneity due to glass formation without localized crystalline defects. A study (Dutta et al 2001) carried out on the corrosion properties of rapidly solidified $\mathrm{Ti}_{47} \mathrm{Cu}_{53}$ and $\mathrm{Ti}_{50} \mathrm{Cu}_{50}$ alloys in acidic chloride environments at $R T$ has indicated that the air formed oxide films and defects introduced in the alloy ribbons during their production influence the corrosion resistance of the alloy ribbons.

Partial crystallinity (Dutta et al 1995; Savalia et al 1996), air pockets (Gravano et al 1992) and compositional difference (Devine and Wells 1976) are the deficiencies those may be introduced in the amorphous alloy ribbons during their production by melt spinning/planar flow casting process. A comparatively slower cooling rate

\footnotetext{
*Author for correspondence
}

of the air side surface than the other side generally induces partial crystallinity in the side. From corrosion point of view, the presence of microcrystalline phases in the amorphous matrix has been reported to be detrimental (Dutta et al 1995; Zhang et al 1991, 1992). Air pockets that mostly exist in the wheel side surface of the ribbon originate from entrapment of air during the production of alloy ribbons by rapid solidification. These air pockets are usually found to be randomly distributed in the wheel side surface with varying size. The adverse effect of quenched-in defects on the corrosion resistance of $\mathrm{Fe}_{34} \mathrm{Ni}_{36} \mathrm{Cr}_{10} \mathrm{P}_{14} \mathrm{~B}_{6}$ amorphous alloy has been reported by some investigators (Gravano et al 1992). The inferior corrosion resistance of the wheel side surface of the ribbon could be attributable to higher concentration of quenched-in defects than the other side due to the higher cooling rate (Gravano et al 1992). The effect of compositional difference on the electrochemical behaviour of the alloy ribbon has been investigated by some researchers (Devine and Wells 1976). According to them, the observed difference in electrochemical behaviour of the alloy ribbon across its thickness is attributed to their compositional difference.

Corrosion studies of rapidly solidified $\mathrm{Ti}-\mathrm{Cu}$ alloys reported in the literature are limited and have been confined to or around equiatomic composition (Naka et al 1978; Dutta et al 2001). Therefore, studies on $\mathrm{Ti}_{42.9} \mathrm{Cu}_{57.1}$ alloy have been presently undertaken since the composition lies on the line composition of peritectic reaction of $\mathrm{Ti}-\mathrm{Cu}$ alloy system. Since the melt spinning process introduces deficiencies in the alloy ribbons, the corrosion behaviour of both the surfaces of the ribbon have been taken into account separately. 


\section{Experimental}

\section{$2.1 \quad$ Alloy making}

The alloy was made using titanium (99.9 wt.\% pure) and copper ( 99.99 wt.\% pure) by arc melting in a purified argon atmosphere. To ensure the homogeneity of the alloy, repeated melting was undertaken. Rapidly solidified ribbons of $\mathrm{Ti}-\mathrm{Cu}$ alloy having the expected composition of $\mathrm{Ti}_{42.9} \mathrm{Cu}_{57.1}$ was produced by a planar flow casting process. The subscript after each element denotes its expected atomic percentage. The planar flow casting technique consisted of impinging a jet of molten alloy on the outer surface of a rotating copper wheel (diameter $0.2 \mathrm{~m}$ and width $0.02 \mathrm{~m}$ ) at a ejection pressure of $0.15 \mathrm{MPa}$. The rotational speed of the wheel was kept at $2000 \mathrm{rpm}$ yielding ribbon thickness of $50 \mu \mathrm{m}$. The ribbon width and its thickness were obtained as $8 \mathrm{~mm}$ and $50 \mu \mathrm{m}$, respectively.

\subsection{Optical microscopy and $X$-ray diffraction}

Optical microscopic and X-ray diffraction (XRD) studies of the alloy ribbons were performed on either side of the ribbon of the alloy in the as-solidified condition.

\subsection{Electron probe microanalysis}

Electron probe microanalysis (EPMA) was done at several locations on either side of the alloy ribbon in the as-solidified condition to find out the compositional difference between the two sides.

\subsection{Electrochemical studies}

To make samples for electrochemical studies, the alloy ribbons were cut into pieces. The dimensions of the samples were $30 \times 8 \mathrm{~mm}$. All samples were mounted using cold setting resin in such a way that only one surface was exposed. Electrical contact was made via a crocodile clip. During the electrochemical studies, precaution was taken so that the clip holding the sample did not come into contact with the solution. By doing so, the exposed surface area of each sample was lower than the mounted surface area. The exposed surface area was kept in the vicinity of $1.5 \mathrm{~cm}^{2}$. Prior to the electrochemical studies, the samples were cleaned with methanol. Potentiostatic anodic polarization of the alloy was conducted in $0.5 \mathrm{M} \mathrm{H}_{2} \mathrm{SO}_{4}+0.5 \mathrm{M} \mathrm{NaCl}$ solution $(\mathrm{pH} \sim 0)$ and $0.01 \mathrm{~N} \mathrm{HCl}$ solution $(\mathrm{pH} \sim 1.9)$ at room temperature $(R T)$ by taking into consideration the wheel side surface and the air side surface of the ribbon separately. In preparing the solutions, analytical reagent (AR) grade and guaranteed reagent (GR) grade chemicals were used. The volume of solution in the electrochemical cell was about $500 \mathrm{ml}$ for each experiment. Prior to the determination of each polarization curve, time was allowed for the stable open circuit potential (OCP) of the sample to be obtained. A saturated calomel electrode (SCE) was used as the reference electrode and platinum was used for the counter electrode. The anodic potential was increased in steps at a rate of $30 \mathrm{mV} / \mathrm{min}$ from the OCP to a maximum potential of about $+650 \mathrm{mV}$ (SCE). The solutions were deaerated with argon gas before and during the corrosion studies. The experiments were repeated to check their reproducibility. After each anodic polarization run, the sample was cleaned and examined by optical microscope.

\section{Results}

\subsection{Surface morphology}

Figures 1 and 2 show the surface morphology of the wheel side and air side of the $\mathrm{Ti}_{42.9} \mathrm{Cu}_{57.1}$ alloy ribbon, respectively in the as-solidified condition. Air pockets with

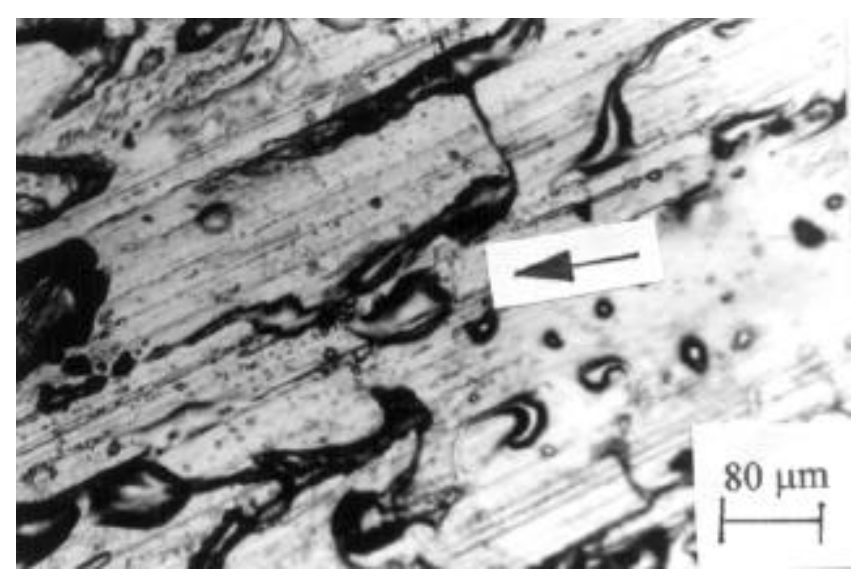

Figure 1. Wheel side surface of rapidly solidified $\mathrm{Ti}_{42.9} \mathrm{Cu}_{57 \cdot 1}$ alloy ribbon revealing air pockets and wheel marks.

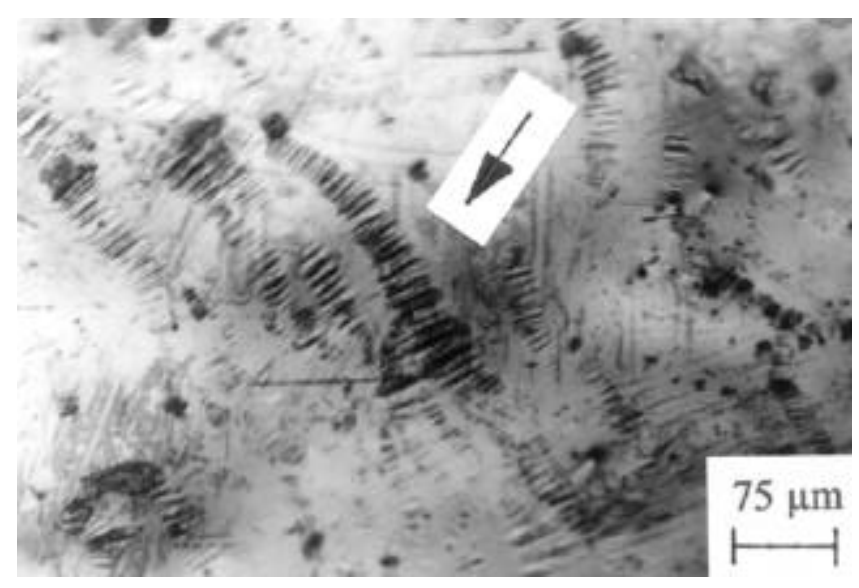

Figure 2. Air side surface of rapidly solidified $\mathrm{Ti}_{42 \cdot 9} \mathrm{Cu}_{57 \cdot 1}$ alloy ribbon revealing dendritic morphology in some locations. 
varying size were found to be randomly distributed in the wheel side surface, whereas air side surface revealed dendritic morphology in some locations. Figures 3 and 4 show X-ray diffractograms of the wheel side and air side surfaces of the alloy ribbon, respectively. The wheel side surface of the alloy ribbon has revealed amorphous structure. The presence of microcrystalline phases in the air side surface of the alloy ribbon could not be detected owing to their small volume fraction.

\subsection{EPMA results}

Table 1 summarizes the results of EPMA for the wheel side and the air side surfaces of the alloy ribbon. The average compositional difference between the two sides of the ribbon has been found to be in the vicinity of 2 atomic percentage. The wheel side surface has been found to be richer in titanium than the other side. The enrichment of one element in one side has been found to be balanced by the depletion of other element in that side.

\subsection{Polarization behaviour}

Figures 5 and 6 show the anodic polarization curves for the $\mathrm{Ti}_{42.9} \mathrm{Cu}_{57.1}$ alloy in $0.5 \mathrm{M} \mathrm{H}_{2} \mathrm{SO}_{4}+0.5 \mathrm{M} \mathrm{NaCl}$ solution and $0.01 \mathrm{~N} \mathrm{HCl}$ solution, respectively. In both the solutions, the alloy displayed passive-pitting behaviour. The passive current density (CD) of the alloy in both the

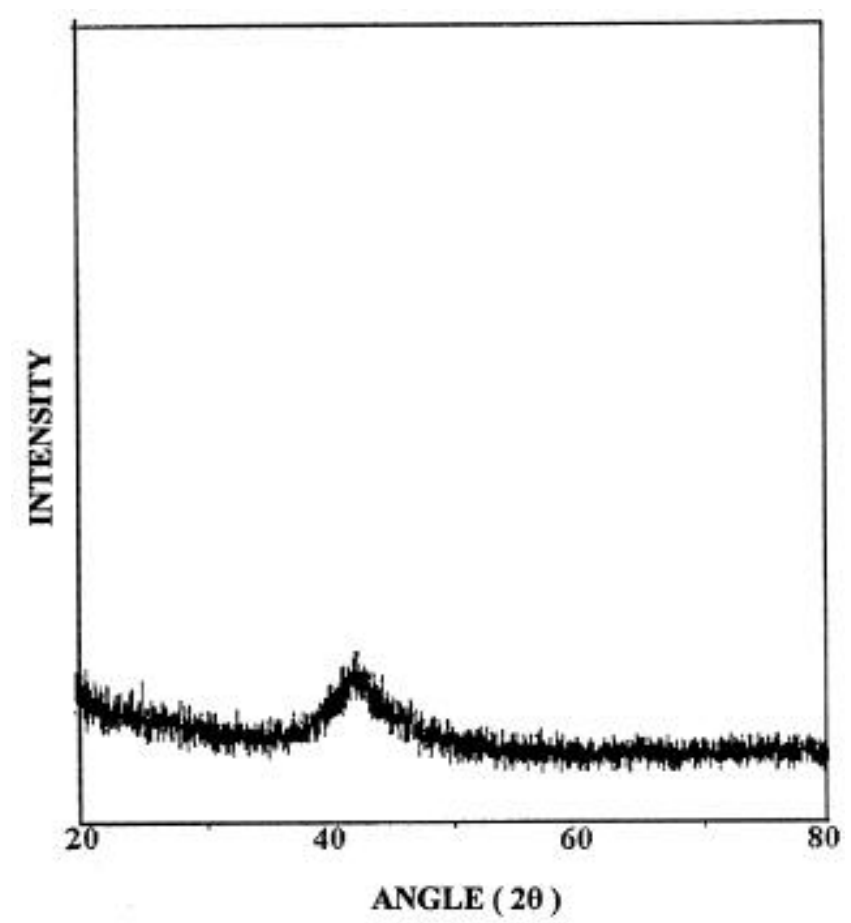

Figure 3. X-ray diffractogram of wheel side surface of rapidly solidified $\mathrm{Ti}_{42.9} \mathrm{Cu}_{57.1}$ alloy ribbon revealing fully amorphous structure. solutions has been found in the vicinity of $0 \cdot 1 \mathrm{~A} / \mathrm{m}^{2}$. In both the solutions, although the nature of the polarization curves for the air side and the wheel side surfaces are similar, the corrosion resistance of the former side has been found to be superior as compared to the latter. The superiority is not only in terms of higher pitting potential, but also in terms of lower anodic CD in the pitting region. In former solution, the superiority of the air side surface is quite pronounced, whereas in latter solution, the superiority is subtle.

\subsection{Pitting of alloy}

The alloy showed pitting after anodic polarization in the acidic chloride environments. Spherical-shaped pits were noticed in either side of the alloy ribbon. Figures $7 \mathrm{a}$ and $\mathrm{b}$ show pitting of the wheel side and air side surfaces of $\mathrm{Ti}_{42.9} \mathrm{Cu}_{57.1}$ alloy ribbon, respectively, after anodic polarization in $0.5 \mathrm{M} \mathrm{H}_{2} \mathrm{SO}_{4}+0.5 \mathrm{M} \mathrm{NaCl}$ solution.

Table 1. EPMA results of alloy at various locations of ribbons (at.\%).

\begin{tabular}{lcc}
\hline $\begin{array}{l}\text { Expected alloy } \\
\text { composition }\end{array}$ & $\begin{array}{c}\text { Compositions } \\
\text { obtained in wheel } \\
\text { side surface }\end{array}$ & $\begin{array}{c}\text { Compositions } \\
\text { obtained in air } \\
\text { side surface }\end{array}$ \\
\hline $\mathrm{Ti}_{42 \cdot 9} \mathrm{Cu}_{57 \cdot 1}$ & $\mathrm{Ti}_{41 \cdot 79} \mathrm{Cu}_{58 \cdot 21}$ & $\mathrm{Ti}_{39 \cdot 82} \mathrm{Cu}_{60 \cdot 18}$ \\
& $\mathrm{Ti}_{42 \cdot 15} \mathrm{Cu}_{57 \cdot 85}$ & $\mathrm{Ti}_{38 \cdot 78} \mathrm{Cu}_{61 \cdot 22}$ \\
& $\mathrm{Ti}_{40 \cdot 42} \mathrm{Cu}_{59 \cdot 58}$ & $\mathrm{Ti}_{38 \cdot 09} \mathrm{Cu}_{61.91}$ \\
\hline
\end{tabular}

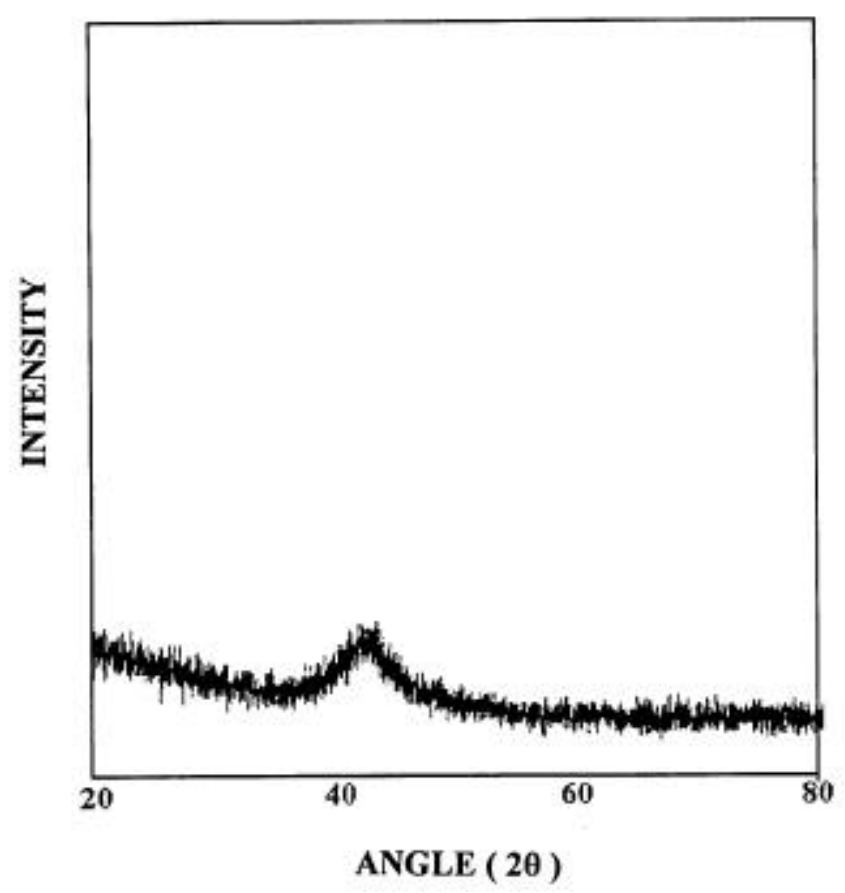

Figure 4. X-ray diffractogram of air side surface of rapidly solidified $\mathrm{Ti}_{42.9} \mathrm{Cu}_{57.1}$ alloy ribbon revealing absence of microcrystalline phases. 


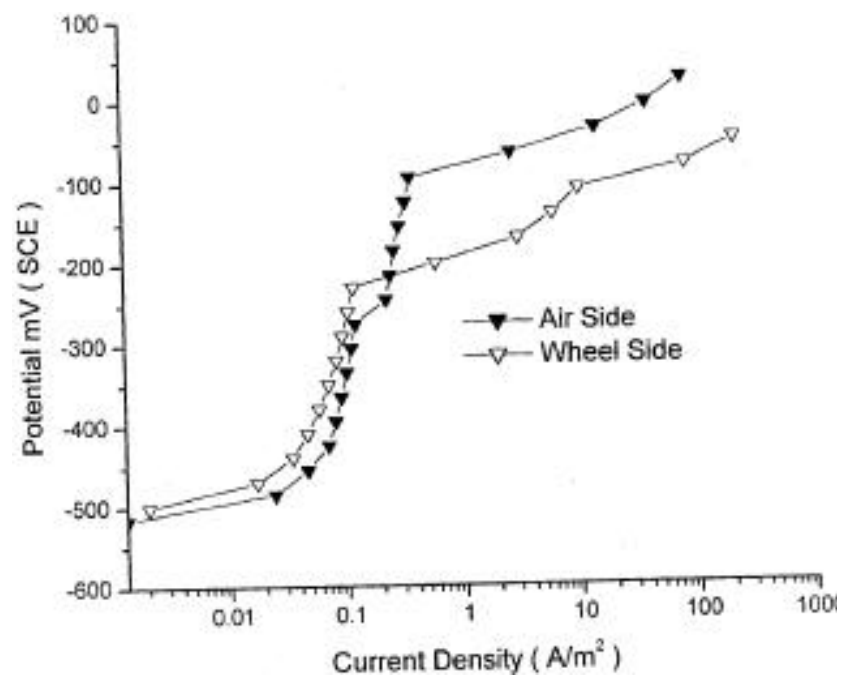

Figure 5. Anodic polarization curves of rapidly solidified $\mathrm{Ti}_{42.9} \mathrm{Cu}_{57.1}$ alloy ribbon in $0.5 \mathrm{M} \mathrm{H}_{2} \mathrm{SO}_{4}+0.5 \mathrm{M} \mathrm{NaCl}$ solution.

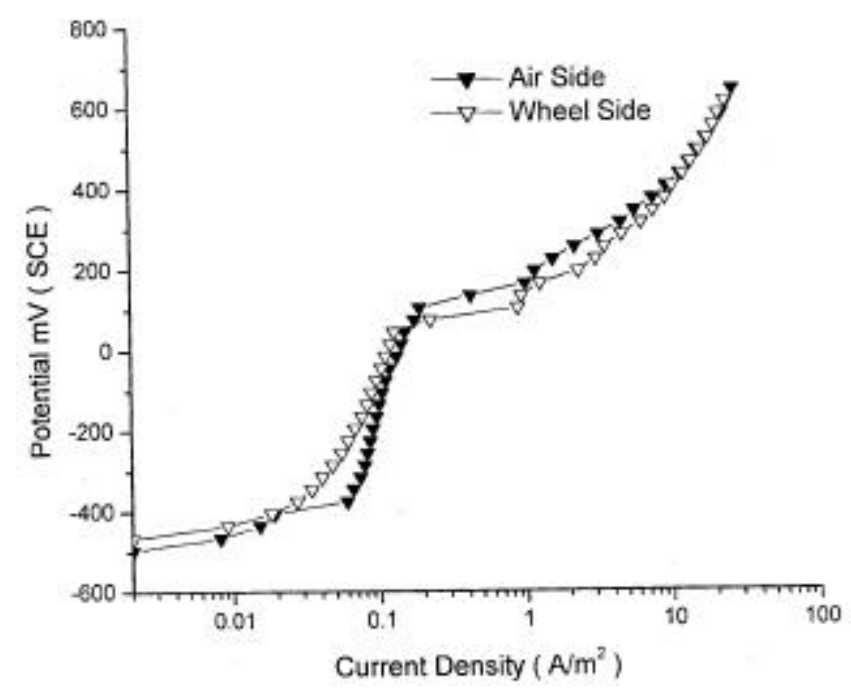

Figure 6. Anodic polarization curves of rapidly solidified $\mathrm{Ti}_{42.9} \mathrm{Cu}_{57.1}$ alloy ribbon in $0.01 \mathrm{~N} \mathrm{HCl}$ solution.

\section{Discussion}

Amorphous materials do not possess defects such as grain boundaries, vacancies and dislocations. Rapid solidification by melt spinning process, widely used for the production of alloy ribbons, may induce deficiencies viz. partial crystallinity, air pockets and compositional difference in the alloy ribbons. Therefore, the corrosion resistance of the alloy ribbons is expected to vary across their thickness. Keeping this in view, an attempt has been made in the present work to correlate the variation in corrosion resistance of the alloy ribbons across its thickness with the three aforementioned deficiencies.

The wheel side surface of the as-solidified $\mathrm{Ti}_{42 \cdot 9} \mathrm{Cu}_{57 \cdot 1}$ alloy has displayed inferior corrosion resistance as compared to that of the air side surface in the acidic chloride
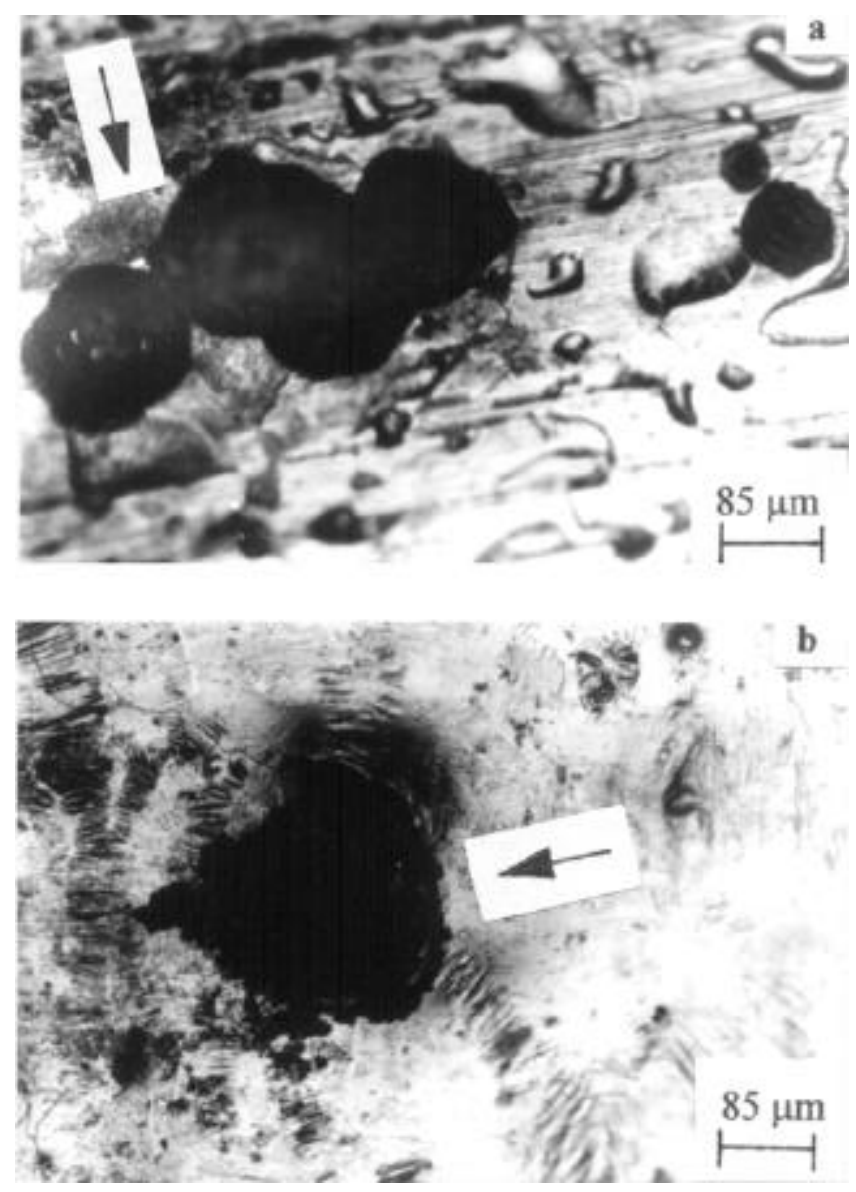

Figure 7. Pitting in (a) wheel side surface and (b) air side surface of rapidly solidified $\mathrm{Ti}_{42.9} \mathrm{Cu}_{57.1}$ alloy ribbon, after anodic polarization in $0.5 \mathrm{M} \mathrm{H}_{2} \mathrm{SO}_{4}+0.5 \mathrm{M} \mathrm{NaCl}$ solution.

solutions. Since the studies of the alloy have been carried out in the as-solidified condition, it would be expected to have an air-formed oxide film on either surface produced during planar flow casting. The thickness of the airformed oxide film would be greater on the air side surface as compared to the other side. Since this air-formed oxide film influences the corrosion resistance of the alloy ribbons only in the initial stages, overall inferior corrosion resistance of the wheel side surface could be primarily attributable to high concentration of air pockets. It is pertinent to mention in this context that the removal of air-formed oxide film that is produced during planar flow casting is possible by electropolishing the ribbons. However, in the present work, it is intended to investigate the corrosion behaviour of the alloy ribbons in the as-solidified condition and that is the reason why, electropolishing of the alloy ribbons prior to corrosion studies was not performed. It is worth mentioning here that after electropolishing, the concentration of air pockets (in the wheel side surface) and the volume fraction of the microcrystalline phases (in the air side surface) too are expected to be reduced with a varying proportion. After dissolution of 
air-formed oxide film occurred, both the sides of the alloy ribbon have shown passivation followed by pitting in the acidic chloride environments. The chloride ions seem to have facilitated the breakdown of the titanium oxide film (Galvele 1978) leading to pitting of the alloy. In the acidic chloride solutions, the two defects existing in the amorphous matrix viz. microcrystalline phases and air pockets, have not only favoured the breakdown of the oxide film on the air and wheel sides, respectively, but also have provided preferential sites for pit initiation, leading to pitting of the alloy as is evident from the microscopic studies. It is mentioned earlier that the wheel side surface of the alloy ribbon possesses inferior corrosion resistance as compared to that of the air side surface. The inferior corrosion resistance of the former side to that of the latter is not only in terms of lower pitting potential, but also in terms of higher anodic $\mathrm{CD}$ in the pitting region. The inferior corrosion resistance of the wheel side surface could be primarily attributable to high concentration of air pockets. The high concentration of air pockets appears to have favoured easier film breakdown by acting as weak sites leading to lower pitting potential of the side. In addition to it, the oxygen entrapped in the air pockets seems to have accelerated material dissolution by consuming the electrons liberated. This acceleration appears to have continued until all the air pockets are dissolved away. The deleterious effect of $\mathrm{Cl}^{-}$ion on the corrosion resistance of rapidly solidified $\mathrm{Ti}_{42.9} \mathrm{Cu}_{57.1}$ alloy as noticed in the present work is in agreement with the findings of others (Naka et al 1978). According to Naka et al, amorphous $\mathrm{Ti}_{50} \mathrm{Cu}_{50}$ alloy is stable in $1 \mathrm{~N} \mathrm{H}_{2} \mathrm{SO}_{4}, 1 \mathrm{~N} \mathrm{HNO}_{3}$ and $1 \mathrm{~N} \mathrm{NaOH}$ solution but unstable in aggressive chloride solutions like $1 \mathrm{~N} \mathrm{HCl}$ and $3 \% \mathrm{NaCl}$.

The deleterious effect of quenched-in defects on the corrosion resistance of $\mathrm{Fe}_{34} \mathrm{Ni}_{36} \mathrm{Cr}_{10} \mathrm{P}_{14} \mathrm{~B}_{6}$ amorphous alloy has been reported by some investigators (Gravano et al 1992). According to them, the inferior corrosion resistance of the wheel side surface of the ribbon has resulted from its higher concentration of quenched-in defects as compared to the other side due to higher cooling rate. In the present work, the inferior corrosion resistance of the wheel side surface as compared to the air side could be primarily attributed to the higher concentration of air pockets in the former side. This finding is in accordance with the findings of others (Gravano et al 1992).

The detrimental effects of microcrystalline phases on the corrosion of amorphous alloys have been reported by some investigators (Dutta et al 1995; Zhang et al 1991, 1992). The presence of microcrystalline phases such as orthorhombic $\mathrm{Zr}_{3} \mathrm{Fe}$, bct $\mathrm{Zr}_{2} \mathrm{Ni}$ and $\mathrm{HCP} \alpha-\mathrm{Zr}$ in the air side surface of rapidly solidified $\mathrm{Zr}_{76} \mathrm{Ni}_{16} \mathrm{Fe}_{8}$ alloy has been reported to be detrimental on the corrosion resistance of the alloy in chloride solutions (Dutta et al 1995). Similar adverse effects of microcrystalline phases like bcc $\mathrm{Cr}(\mathrm{Ni}, \mathrm{P})$ and $\mathrm{CrNi}_{3} \mathrm{P}$ in the amorphous matrix of melt-spun $\mathrm{Cr}-27 \mathrm{Ni}-15 \mathrm{P}$ alloy have been discussed by other researchers (Zhang et al 1991). According to them (Zhang et al 1991, 1992), those microcrystalline phases considerably reduce the corrosion resistance of the alloy in $6 \mathrm{M} \mathrm{HCl}$ solution. In the present work, optical microscopy on the air side surface of rapidly solidified $\mathrm{Ti}_{42.9} \mathrm{Cu}_{57.1}$ alloy ribbon has revealed the presence of microcrystalline phases in the amorphous matrix. The presence of these microcrystalline phases has been found to be harmful from the viewpoint of initiating oxide film breakdown and pitting.

Some investigators (Devine and Wells 1976) have noticed a difference in electrochemical behaviour for the alloy ribbon across its thickness. According to them, the difference in electrochemical behaviour between the two sides of the ribbon is attributable to compositional difference. In the present work, the average compositional difference between the two sides of the ribbon of rapidly solidified $\mathrm{Ti}_{42.9} \mathrm{Cu}_{57.1}$ alloy has been found to be in the vicinity of 2 atomic percentage. This difference is believed to have an insignificant role on the corrosion behaviour of the ribbon surfaces (Dutta et al 2001).

\section{Conclusions}

The following conclusions can be drawn from the present work.

(I) Partial crystallinity, air pockets and compositional difference are the three deficiencies introduced in the ribbons of rapidly solidified $\mathrm{Ti}_{42 \cdot 9} \mathrm{Cu}_{57 \cdot 1}$ alloy.

(II) The corrosion resistance of the ribbons in the acidic chloride environments varies across their thickness due to the introduction of defects.

(III) The wheel side surface of the ribbon possesses inferior corrosion resistance as compared to the air side surface.

(IV) Air pockets seem to be the most deleterious defect from the viewpoint of corrosion resistance of the alloy ribbons.

\section{Acknowledgements}

The authors express their gratitude to Dr S Banerjee for his encouragement during the course of the study. It is also a pleasure to acknowledge the benefits of a number of discussions the authors had with Dr P K De, Dr P Mukhopadhyay and Dr E G Baburaj during the course of the study. The authors acknowledge the help provided by Dr G B Kale and Dr C N Rao in carrying out EPMA and XRD, respectively of the samples. Thanks are due to Mrs P Agashe for assistance in photomicrography. 


\section{References}

Devine T M and Wells L 1976 Scr. Metall. 10309

Dutta R S, Savalia R T and Dey G K 1995 Scr. Mater. 32 207

Dutta R S, Savalia R T and Dey G K 2001 Br. Corr. J. 36 221

Galvele J R 1978 Passivity of metals (eds) R P Frankenthal and J Kruger (Princeton, NJ: The Electrochemical Society) p. 285
Gravano S M, Torchio S, Mazza F, Angelini E and Baricco M 1992 Corr. Sci. 331227

Naka M, Hashimoto K and Masumoto T 1978 J. Non-Cryst. Solids $\mathbf{3 0} 29$

Savalia R T, Tewari R, Dey G K and Banerjee S 1996 Acta Mater. 4457

Zhang B-P, Habazaki H, Kawashima A, Asami K, Hiraga K and Hashimoto K 1991 Corr. Sci. 32433

Zhang B-P, Habazaki H, Kawashima A, Asami K and Hashimoto K 1992 Corr. Sci. 33103 